\title{
Adaptive optical network unit for point-to-point and point-to-multipoint Gigabit Ethernet-based optical access networks
}

\author{
Junichi Kani ${ }^{a)}$, Ryogo Kubo, Mitsumasa Okada, \\ Yukihiro Fujimoto, and Kiyomi Kumozaki \\ NTT Access Network Service Systems Laboratories, NTT Corporation, \\ 1-6 Nakase, Mihama-ku, Chiba, 262-0023 Japan \\ a)kani.junichi@ansl.ntt.co.jp
}

\begin{abstract}
This paper proposes and demonstrates an optical network unit (ONU) that is adaptive in both point-to-point (P2P) and point-tomultipoint (P2MP) optical access networks (OANs) based on Gigabit Ethernet. Use of the proposed ONU makes it possible for network operators to reduce their operational expenditure (OPEX) by controlling/managing necessary inventories as well as handling troubles due to ONU mis-connection.
\end{abstract}

Keywords: optical access, passive optical network, Gigabit Ethernet Classification: Photonics devices, circuits, and systems

\section{References}

[1] H. Shinohara, "Broadband Access in Japan: Rapidly Growing FTTH Market," IEEE Commun. Mag., pp. 72-78, Sept. 2005.

[2] IEEE standard 802.3.

[3] T. Tatsuta, N. Oota, N. Miki, and K. Kumozaki, "Design philosophy and performance of a GE-PON system for mass deployment," J. Opt. Netw., vol. 6, no. 6, pp. 689-700, June 2007.

[4] J. Kani, K. Iwatsuki, and T. Imai, "Optical multiplexing technologies for access-area applications," IEEE J. Sel. Top. Quantum Electron., vol. 12, pp. 661-668, 2006.

[5] K. McCammon and S. W. Wong, "Experimental Validation of an Access Evolution Strategy: Smooth FTTP Service Migration Path," The National Fiber Optic Engineers Conference (NFOEC) 2007, NThB3, 2007.

\section{Introduction}

To cope with the explosive bandwidth demands in Internet access services, network operators have started to massively deploy optical access networks (OANs), for which either point-to-point (P2P) or point-to-multipoint (P2MP) optical access is employed between a central office and end users depending 
on the requirements. While the application area of $\mathrm{P} 2 \mathrm{P}$ optical access includes apartments and business users, P2MP optical access based on passive optical networks (PONs) provides broad bandwidth for residential users in a cost effective manner [1]. In particular, Gigabit-class OANs are popular for both $\mathrm{P} 2 \mathrm{P}$ and $\mathrm{P} 2 \mathrm{MP}$ optical access given that current applications provide broadband services with maximum speeds from $100 \mathrm{Mbit} / \mathrm{s}$ to $1 \mathrm{Gbit} / \mathrm{s}$. As for OANs based on Gigabit Ethernet, IEEE standard has both P2P and P2MP specifications [2]; the Gigabit Ethernet-based P2MP OAN is known as GE-PON [3].

Operating/maintaining two different systems (i.e. P2P and P2MP) potentially incurs relatively high operational expenditure (OPEX) for network operators in terms of controlling/managing inventories of optical network units (ONUs) needed as well as handling troubles caused by connecting the wrong type of ONU. The latter includes possible PON outage due to misconnecting a P2P ONU to a branch of a P2MP OAN; every P2P ONU continuously generates optical signals, which potentially interferes with the optical signals emitted from the valid P2MP ONUs.

To address these issues, this paper proposes an adaptive ONU that can, in a simple manner, accommodate both P2P and P2MP Gigabit Ethernetbased OANs. The adaptive ONU makes it possible for network operators to reduce their OPEX since it simplifies inventory management as well as eliminates the problem of ONU mis-connection.

\section{Concept}

Fig. 1 shows the application of the proposed adaptive ONUs for $\mathrm{P} 2 \mathrm{P}$ and P2MP OANs; a normal P2P optical line terminal (OLT) and PON OLT are used in the central office $(\mathrm{CO})$ for $\mathrm{P} 2 \mathrm{P}$ and P2MP OANs, respectively. The adaptive ONU recognizes the OLT type by monitoring the downstream signal via fiber when it starts working, and then starts communication with the protocol appropriate to the OLT type. Fig. 1 also indicates that P2P OANs can be evolved into wavelength-division multiplexing PON (WDM-PON); a number of P2P links are multiplexed into a single fiber via WDM [4]. Such

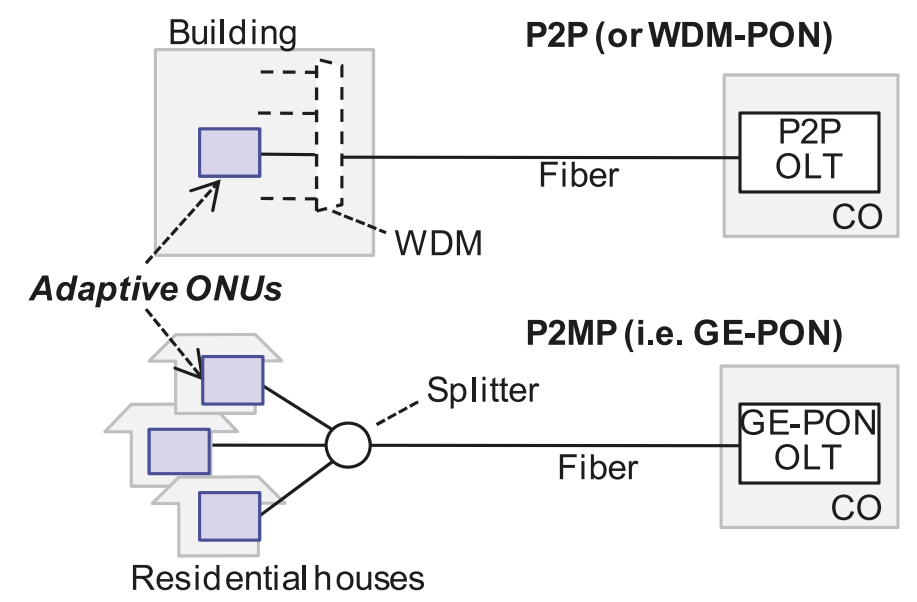


a WDM extension has been studied for G-PON as well [5]. The adaptive ONUs can accommodate such WDM extensions by using pluggable or tunable transceivers.

An alternative approach to integrate $\mathrm{P} 2 \mathrm{P}$ and $\mathrm{P} 2 \mathrm{MP}$ OANs is to use a P2MP OLT (i.e. GE-PON OLT) instead of a P2P OLT in P2P OANs to make it possible to use P2MP ONUs (i.e. GE-PON ONUs) in both P2P and P2MP OANs. The cost of the PON OLT can be potentially decreased in part by using $\mathrm{P} 2 \mathrm{P}$-specific optics. While this approach may be useful for green-field deployment, depending on the cost decrease of the P2MP OLT, replacement of all $\mathrm{P} 2 \mathrm{P}$ OLTs is required in existing OANs. Therefore, the rest of this paper focuses on the adaptive-ONU approach.

\section{Principle}

The simple principle of the adaptive ONU is to discriminate the downstream signal type (P2P or P2MP) by checking the position of the start-of-frame delimiter (SFD) and to switch the main logic according to the signal type. SFD is located at the 8th octet in the preamble in the case of P2P (i.e. normal Gigabit Ethernet) while it is at the 3rd octet in the case of P2MP (i.e. GE-PON) as shown in Fig. 2 (a) [2]. In addition, P2MP OLTs frequently send multi-point control signals while typical P2P OLTs may send only the idle pattern or the auto-negotiation signal when not connecting to ONU(s). Because both the idle pattern and auto-negotiation signal (for 1000BASE-X) are encoded with 8B10B [2], an ONU can first recognize that the equipment on the other side of the fiber is either a Gigabit Ethernet-based P2P OLT or a GE-PON OLT by checking the correct 8B10B decode. Second, the ONU can distinguish if it is a P2P OLT or a GE-PON OLT by checking existence of Ethernet frame(s) for a period (e.g. 1s) as well as checking the position of SFD if frames are detected.

Fig. 2 (b) shows an example of the block diagram of the adaptive ONU.

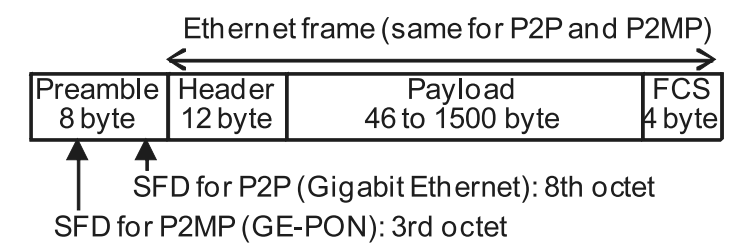

(a) SFD position in preamble of Ethernet frames

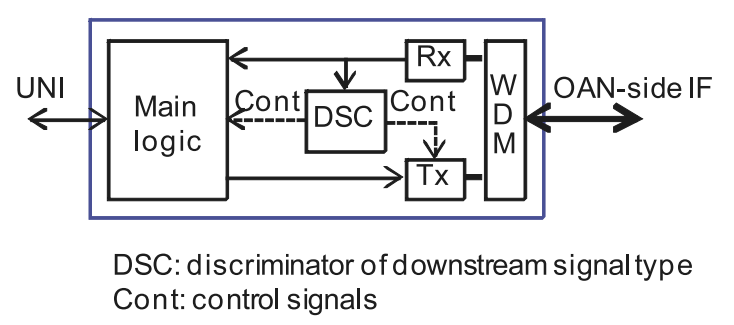

(b) An example of the ONU block diagram

Fig. 2. Principle of the adaptive ONU. 
The main logic has two modes, one is P2P (i.e. normal Gigabit Ethernet) mode and the other is P2MP (i.e. GE-PON mode), but the difference is simple; P2MP logic can basically functions as the P2P mode by adjusting the SFD position as well as disabling the functions of the multipoint MAC control protocol (MPCP) [2]. The discriminator (DSC) not only controls the mode of the main logic but also controls the transmitter (Tx), which is set disabled before DSC discriminates the signal type.

We note that the transmitting/receiving optical-power specifications for P2P ONUs are lower/higher than those for P2MP ONUs as per the IEEE 802.3 standard because the maximum loss budget of P2P OAN is smaller than that of P2MP OAN. However, an additional optical-power adjustment function to deal with this difference is not studied in this paper because an adaptive ONU with P2MP optics can cover the case of a smaller loss budget in practice.

\section{Demonstration}

We demonstrated the feasibility of the adaptive ONU in an experiment where the DSC was realized by a fully programmable gate array (FPGA) and the main logic was simulated by using a P2P and GE-PON logics in parallel: the experimental setup is shown as Fig. 3 (a). The FPGA was programmed to generate GE-PON and P2P detection signals, each was 2-level signal, to show the discrimination result. The FPGA controlled the optical transceiver (TRx), which was set disabled before DSC discriminated the signal type. An optical transceiver intended for GE-PON ONUs was used as TRx. The FPGA was also programmed to reenter the initial state and thus disable the TRx whenever continuous 8B10B decode errors were detected.

Time-intensity charts of (1) downstream, (2) GE-PON detection, (3) P2P detection and (4) upstream signals were gathered for two cases; in one, the adaptive ONU was connected to a P2P OLT (case 1) and in the other it was connected to a GE-PON OLT (case 2). In case 2, a normal GE-PON ONU was connected to the OLT in addition to the adaptive ONU via a 1-by-8 optical splitter as shown in Fig. 3 (a), which was replaced by an attenuator in case 1.

Fig. 3 (b) and (c) show results of the time-intensity measurements in case 1 and case 2, respectively. As can be seen, the adaptive ONU successfully started to operate as a P2P ONU and a GE-PON ONU when connected to the P2P OLT and the GE-PON OLT, respectively. We also confirmed that the adaptive ONU stopped emitting upstream signals immediately when the downstream signal was stopped. In case 1, the ONU took 1 second to discriminate the downstream-signal type as shown in Fig. 3 (b). This period was that set in the FPGA to check the existence of Ethernet frame(s) as explained in the previous section. In case 2, the ONU took around 3 seconds to start sending upstream signals as shown in Fig. 3 (c). This was due to the authentication needed for the PON logic. 


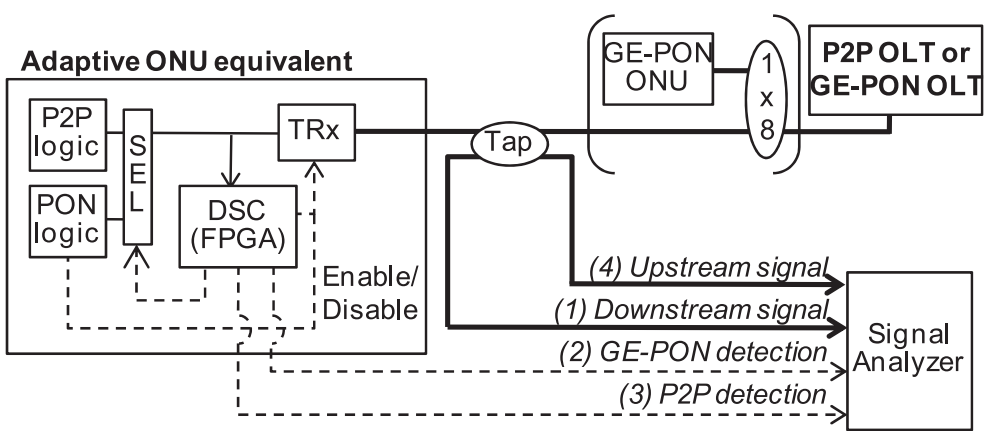

DSC: Discriminator of d ownstream signal, TRx: Optical Transceiver, SEL: Selector, 1x8: 1-by-8 optical splitter

(a)

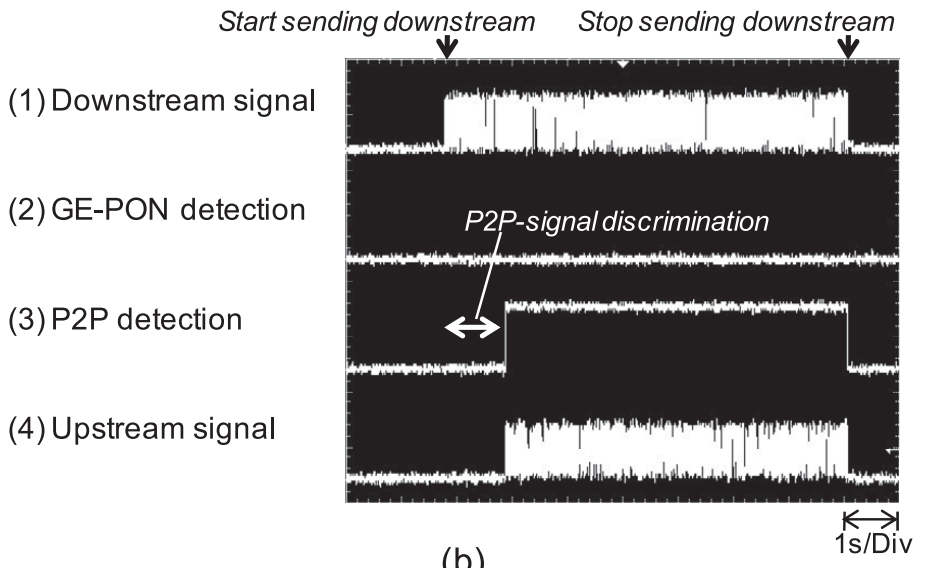

(b)

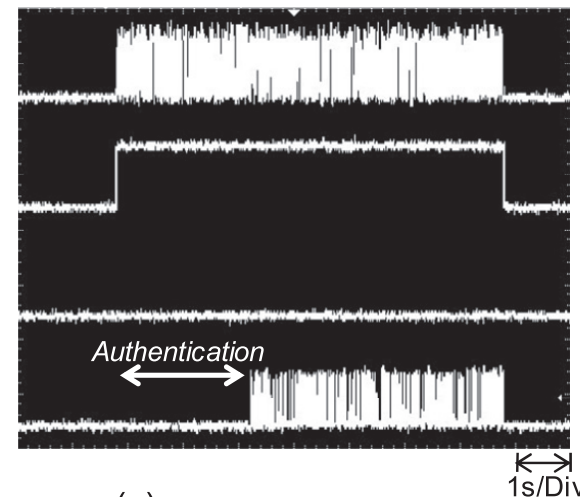

(c)

Fig. 3. Experiment to measure time-intensity charts: (a) setup, (b) result when connected to a P2P OLT, and (c) result when connected to a GE-PON OLT.

\section{Conclusion}

We proposed the adaptive ONU concept as well as its principle; it reduces the operator's operational burdens by reducing the inventories needed as well as eliminating the risk associated with ONU mis-connection in point-to-point and point-to-multipoint Gigabit Ethernet-based OANs. Its feasibility was successfully demonstrated through an experiment. 